\title{
Gate insulators at the limit
}

\begin{abstract}
The development of competitive field-effect transistors based on two-dimensional semiconductors will also require the development of suitably scaled insulators.
\end{abstract}

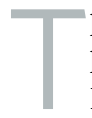

he field-effect transistor lies at the heart of the electronics industry. In such devices, the flow of current through a conductive channel, formed between source and drain electrodes, is controlled by a voltage applied to a gate electrode. Two-dimensional (2D) materials - from graphene and phosphorene to molybdenum disulfide $\left(\mathrm{MoS}_{2}\right)$ and tungsten diselenide $\left(\mathrm{WSe}_{2}\right)$ - currently sit at the forefront of efforts to replace silicon as the channel material, and create transistors with ever smaller dimensions. But the channel in the devices needs to be separated from the gate electrode by an insulating layer, known as the gate dielectric. And the development of competitive devices based on $2 \mathrm{D}$ materials will also require the development of suitably scaled gate dielectrics.

One advantage of silicon is that it has a native oxide - silicon dioxide $\left(\mathrm{SiO}_{2}\right)$ - that can act as a gate dielectric. When very thin (around 5 atoms), gate leakage current due to quantum tunnelling becomes a factor for silicon dioxide, and thus materials with a high dielectric constant - known as high- $\kappa$ dielectrics - have also been developed ${ }^{1}$. Intel, for example, began using hafnium oxide $\left(\mathrm{HfO}_{2}\right)$ back in $2007^{2}$. However, both silicon dioxide and hafnium oxide are amorphous when grown in thin layers and creating an appropriate interface with $2 \mathrm{D}$ channel materials is problematic. In pursuit of $2 \mathrm{D}$ devices, researchers have thus turned to $2 \mathrm{D}$ insulators.

Hexagonal boron nitride (hBN) is the most commonly used 2D insulator. The material is crystalline and can form a clean van der Waals interface with other 2D materials. (It also has the unusual distinction that two researchers - Takashi Taniguchi and Kenji Watanabe at the National Institute of Materials Science (NIMS) in Tsukuba - supply much of the material used in labs across the world ${ }^{3}$.) In a Perspective article in this issue of Nature Electronics, Theresia Knobloch, Tibor Grasser and colleagues consider the performance limits of hexagonal boron nitride.

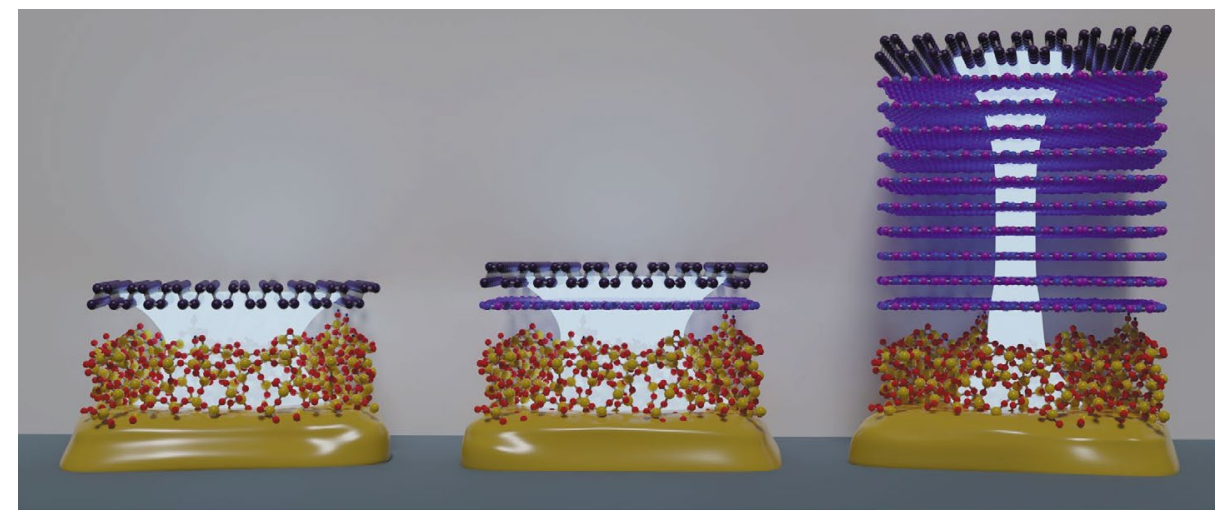

Schematic showing layers of hexagonal boron nitride (boron, pink; nitrogen, blue) at the interface between black phosphorus (phosphorus, dark purple) and silicon dioxide (silicon, yellow; oxygen, red). Figure reproduced from the Perspective article by T. Knobloch et al.

The researchers - who are based at TU Wien in Vienna, the Ioffe Institute in St Petersburg, ETH Zürich, NIMS, and KAUST in Thuwal - compare, in particular, the leakage currents of thin layers of hexagonal boron nitride with that of other $2 \mathrm{D}$ gate insulators. They also consider the ideal case of defect-free hexagonal boron nitride. The analysis leads them to conclude that "even in the most optimistic case, hBN is unlikely to be a good choice for a gate insulator in nanoscaled $2 \mathrm{D}$ complementary metaloxide-semiconductor (CMOS) logic."

There are though alternative insulators. Grasser and colleagues at TU Wien have, for instance, previously reported that epitaxial calcium fluoride $\left(\mathrm{CaF}_{2}\right)$, which forms a quasi van der Waals interface with $2 \mathrm{D}$ semiconductors, can act as a thin gate insulator for 2D devices, building bilayer molybdenum disulfide field-effect transistors with calcium fluoride layers that are around $2 \mathrm{~nm}$ thick ${ }^{4}$. Alternatively, thin layers of hafnium oxide have been deposited on $2 \mathrm{D}$ semiconductors using a molecular crystal as a buffer layer ${ }^{5}$. It is also possible to have $2 \mathrm{D}$ semiconductors (such as bismuth oxyselenide; $\mathrm{Bi}_{2} \mathrm{O}_{2} \mathrm{Se}$ ) that can form a native high- $\kappa$ oxide ${ }^{6}$.

Knobloch, Grasser and colleagues highlight five characteristics for an ideal gate insulator for devices based on 2D materials: small gate leakage currents, excellent gate control, low defect densities, high breakdown strength and optimized mobility in the semiconductor. Their analysis shows that there are a number of materials that can offer lower leakage currents in scaled layers than hexagonal boron nitride. But what material can address all the necessary requirements remains an open question and the search for a suitable gate insulator goes on.

Published online: 23 February 2021 https://doi.org/10.1038/s41928-021-00553-5

\footnotetext{
References

1. Bohr, M. T., Chau, R. S., Ghani, T. \& Mistry, K. IEEE Spectrum 44, 29-35 (2007).

2. Intel's fundamental advance in transistor design extends Moore's law, computing performance. Intel News Release https://go.nature. com/36GYZH1 (11 November 2007).

3. Zastrow, M. Nature 572, 429-432 (2019).

4. Illarionov, Y. Y. et al. Nat. Electron. 2, 230-235 (2019).

5. Li, W. et al. Nat. Electron. 2, 563-571 (2019).

6. Li, T. et al. Nat. Electron. 3, 473-478 (2020).
} 